\title{
Territory and Sustainable Economic Development in Morocco
}

\section{Mina Elgraini}

\author{
Mohammed V Souissi University, Rabat, Morocco.
}

\section{Doi:10.5901/mjss.2013.v4n10p699}

\section{Abstract}

The magnitude of the challenges that are facing Morocco and that are becoming more pressing as the effects of globalization and the opening up of economies argues for the introduction of new concepts and procedures for territories development that combine progress and social justice, economic efficiency and development of natural resources for sustainable development. The latter can not be guaranteed with the participation and the involvement of all local and regional actors in the design of their territorial development projects. Indeed, while the concept and objectives of sustainable development are universal in nature, the application can only be differentiated according to the areas studied. In Morocco, the magnitude of the challenges that are becoming increasingly pressing as the effects of globalization and the opening up of economies argues for the introduction of new concepts and procedures for regional development that combine progress and social justice, economic efficiency and development of natural resources for sustainable development. The latter can not be guaranteed with the participation and involvement of all local and regional actors in the design of their territorial development projects. Our research intends to discuss issues of sustainable development and its necessary local roots. It is twofold: firstly, to show that a sustainable and equitable development involves the creation of competitive territories, generating wealth and protector of natural resources, on the other hand, emphasize the policy initiated by Morocco in order to sustainable development at local level.

Keywords: sustainable development, territorial development, Local Productive Systems, Local Agenda 21, Oasis Tafilalet.

\section{Introduction}

Le développement durable commence aujourd'hui à susciter une certaine curiosité bienveillante. C'est en particulier le cas dans les domaines de l'aménagement du territoire où l'on constate que cette préoccupation est progressivement intégrée ; souvent sous la pression des réalités de terrain. En effet, la réflexion sur le développement territorial ne peut faire l'impasse d'une réflexion en termes d'équité et d'efficacité et tout un ensemble de raisons militent a priori pour donner progressivement aux territoires une place privilégiée dans les stratégies futures de développement durable.

Ainsi, si le concept et les objectifs de développement durable sont par nature universels, leur application ne peut être que différenciée en fonction des terrains étudiés. Au Maroc, l'ampleur des défis qui deviennent de plus en plus pressants sous les effets de la mondialisation et de l'ouverture des économies milite pour l'introduction de nouvelles notions et de nouveaux modes opératoires en matière de développement des territoires qui allient progrès et justice sociale, efficacité économique et valorisation des ressources naturelles pour un développement durable. Ce dernier ne peut être garanti qu'avec la participation et l'implication de tous les acteurs locaux et régionaux dans la conception de leurs projets de développement territorial.

Notre recherche se propose de discuter des enjeux du développement durable et son nécessaire ancrage territorial. Elle poursuit un double objectif : d'une part, montrer qu'un aménagement durable et équitable implique la création de territoires compétitifs, générateur de richesse et protecteur des ressources naturelles; d'autre part, souligner la politique initiée par le Maroc afin d'assurer un développement durable au niveau des territoires.

\section{Territoire et développement durable}

Le développement durable n'est pas un concept fermé, forgé définitivement: c'est une stratégie en formation, notamment en rapport avec l'action. Cette vision du Développement Durable a forcément des dimensions territoriales variées. En effet, consacré par le Sommet de la Terre de Rio en 1992, le développement durable est aujourd'hui inscrit dans les orientations politiques nationales et internationales. Mais c'est bien à l'échelle des territoires que se dessine depuis quelques années un renouvellement de l'action, inscrivant concrètement le développement durable dans les réalités sociales et économiques. 
S'attaquer aujourd'hui de manière conjointe à l'aménagement du territoire et au développement durable est justifié car les difficultés de ces deux domaines ont quelques rapports et on peut envisager des actions de réciprocité des impacts positifs.

\subsection{Concept de développement durable}

C'est dans un climat de confrontation et non de conciliation entre l'écologie et l'économie que se tient la Conférence des Nations Unies sur l'Environnement humain, à Stockholm, en 1972. Conférence qui sera à l'origine du premier vrai concept de développement durable, baptisé à l'époque éco-développement. On a insisté sur la nécessité d'intégrer l'équité sociale et la prudence écologique dans les modèles de développement économique du Nord et du Sud. II en découlera la création du Programme des Nations Unies pour l'Environnement (PNUE) ainsi que le Programme des Nations Unies pour le Développement (PNUD).

Le concept de développement durable est apparu, au sein de l'Union Internationale pour la Conservation de la Nature (UICN) au début des années 80. II a été repris et popularisé par le rapport Brundtland "Notre avenir à tous" (CMED, 1989) publié par la Commission mondiale sur l'environnement et le développement et a servi de référence lors du Sommet mondial de Rio De Janeiro en 1992.

Le concept est donc issu d'un long processus de réflexion lors des négociations internationales menées au sein de l'ONU. Ainsi, la Commission mondiale sur l'environnement et le développement a défini le développement durable comme suit (Burgenmeier, 2007) : « Un processus de changement par lequel l'exploitation des ressources, l'orientation des investissements, des changements techniques et institutionnels se trouvent en harmonie et renforcent le potentiel actuel et futur de satisfaction des besoins des hommes ».

Le point de départ est l'inquiétude de la commission devant les signes de " grave stress subi par les systèmes économiques, environnementaux et sociaux partout dans le monde ». II s'agit d'associer des considérations environnementales et économiques aux processus décisionnels. La commission postule ainsi que le développement à long terme n'est viable qu'en conciliant le respect de l'environnement, l'équité sociale et la rentabilité économique (Férone,G., Debas,D., \& Genin,A.S., 2004).

Ainsi, le développement doit être général, au sens de la valorisation de l'ensemble des atouts d'un territoire, et équilibré, au sens d'une maîtrise des interactions entre les facteurs et les processus, et intégrer la notion de temporalité : le temps à venir ( "satisfaire les aspirations des générations futures »), comme le temps présent (" ne pas sacrifier pour autant les aspirations de la génération actuelle »), et prendre en considération autant l'influence du monde extérieur sur un territoire donné, que la contribution de chaque territoire à l'équilibre de l'écosystème global. Un principe qui renvoie à la notion de co-responsabilité (Vandermotten, C., 2002).

Le développement durable a aujourd'hui dépassé le stade du simple concept. II constitue désormais un véritable enjeu de responsabilisation des organisations qui sont confrontées à des défis d'équité sociale, d'efficacité économique et de préservation de l'environnement. Le développement durable se veut un processus de développement qui concilie l'écologique, l'économique et le social et établit un cercle vertueux entre ces trois pôles: c'est un développement, économiquement efficace, socialement équitable et écologiquement soutenable.

La question du développement durable implique de nouvelles attitudes et responsabilités qui vont au-delà de l'environnement et concernent désormais le rôle social et sociétal de l'entreprise. Selon cette perspective, les domaines de la justice sociale (équité), de la diversité, de la solidarité intergénérationnelle, de l'aménagement équilibré de l'espace, du dialogue social ( meilleure relation avec les collaborateurs de l'entreprise, négociation collective) ou encore de la citoyenneté ( responsabilité sociale, politique de transparence, réputation et fidélisation, relation constructive avec la communauté locale et les acteurs sociaux) font désormais l'objet d'enjeux stratégiques, en positionnant ces éléments au cœur de la politique générale des groupes ( Dion.M.,Wolf.D. et al, 2008)

Le développement durable repose en fait sur une nouvelle forme de gouvernance, où la mobilisation et la participation de tous les acteurs de la société civile aux processus de décision doit prendre le pas sur le simple échange d'informations. Le développement durable entend promouvoir la démocratie participative et rénover l'approche citoyenne. L'accès à l'information, et la transparence en sont les pré-requis.

Aucun acteur ou responsable, tant au niveau individuel que collectif, ne peut envisager une quelconque stratégie sans faire référence à sa « durabilité ». Le développement durable est au cœur de tous les discours officiels et, chaque jour, des experts, toutes disciplines confondues, se réunissent pour en débattre. On peut donc légitimement s'interroger sur la relation qui existe entre l'aménagement du territoire et le développement durable. 


\subsection{L'agenda 21 local : projet territorial de développement durable}

L'aménagement et le développement durables des territoires sont aujourd'hui au cœur des préoccupations quotidiennes de l'action publique. Les défis à relever collectivement sont nombreux, qu'il s'agisse de garantir la cohésion sociale, à travers notamment les politiques de l'habitat, de renforcer l'attractivité des territoires ou d'assurer l'intégration de l'environnement dans les projets d'aménagement.

Si l'échelle territoriale apparaît bien a priori comme un point d'appui privilégié des démarches de "développement durable" il est incontestable que c'est à l'échelle des territoires que le "développement durable" a été le plus rapidement et visiblement intégré dans les politiques et ceci sous les formes les plus diverses. En effet, les agendas 21 locaux, se basant sur une philosophie de l'action (Pierron.J.P., 2009), sont devenus la traduction la plus immédiate de « l'intégration » du développement durable dans les politiques publiques.

L'Agenda 21 (ou Action 21) est un plan d'action pour le 21ème siècle adopté par 173 chefs d'État lors du sommet de la Terre, à Rio de Janeiro, en 1992. Avec ses 40 chapitres, ce plan d'action décrit les secteurs où le développement durable doit s'appliquer dans le cadre des collectivités territoriales.

Cet outil permet d'associer l'ensemble des acteurs interne et externe à la collectivité par une véritable sensibilisation aux enjeux actuels de développement durable. C'est donc un projet territorial de développement durable qui se veut :

$\checkmark$ participatif, mobilisant différents acteurs impliqués sur le territoire,

$\checkmark$ volontaire, à l'initiative des collectivités,

$\checkmark$ transversal, coordonnant les différents enjeux, les différentes compétences et les différents niveaux de collectivités,

$\checkmark$ adapté aux spécificités du territoire,

$\checkmark$ articulant une logique de long terme à des actions pragmatiques et concrètes.

Les collectivités territoriales sont appelées, dans le cadre du chapitre 28 de l'Agenda 21 de Rio, à mettre en place un programme d'Agenda 21 à leur échelle, intégrant les principes du développement durable, à partir d'un mécanisme de consultation de la population : ainsi naît l'Agenda 21 local. « Elles jouent, au niveau administratif le plus proche de la population, un rôle essentiel dans l'éducation, la mobilisation et la prise en compte des vues du public en faveur d'un développement durable » (Extrait du chapitre 28).

L'Agenda 21 local est qualifié d'une innovation sociale, appliquée à l'aménagement durable et conséquemment à la gestion du territoire (Gagnon, C., 2007), puisqu'il s'agit :

$\checkmark$ D'une transformation sociale dans la façon de penser et de faire le développement tant sur le plan des énoncés et de la gestion des politiques/actions publiques et privées ;

$\checkmark$ d'une nouvelle gouvernance territoriale, caractérisée d'une part, par une complémentarité entre la démocratie élective et participative, et, d'autre part, par la responsabilisation de tous les acteurs territoriaux ;

$\checkmark$ d'une meilleure transparence dans le mode décisionnel concernant le territoire et sa gestion;

$\checkmark$ d'une vision commune et mobilisatrice du territoire ;

$\checkmark$ d'une démarche en continu du territoire apprenant.

Le programme souligne le rôle déterminant des collectivités locales qui par leurs politiques influent directement sur l'économie, le social et l'environnement et qui par leur proximité à la population peuvent agir en faveur de l'information, l'éducation et la mobilisation en faveur du développement durable.

Des pratiques intéressantes, innovantes se développent un peu partout dans le monde, au niveau régional ou local. Elles témoignent d'une certaine vitalité à l'échelle locale, d'une mobilisation active sur le terrain en faveur d'un développement durable. Au Maroc, de nombreuses initiatives ont vu récemment le jour dans ce sens. L'objectif étant d'inscrire le pays dans une démarche de développement durable en assurant la préservation des atouts différenciateurs du Maroc : sa nature, ses populations et ses valeurs, et sa culture et ses traditions.

\section{Territoire et développement économique durable au Maroc}

Le développement durable ne peut devenir réalité qu'à partir du moment où il est approprié par les territoires et surtout par les acteurs de ces territoires. S'agissant d'un mouvement d'envergure internationale, le Maroc a compris cette exigence et l'a traduite par des pratiques innovantes. Cette partie vise à positionner le Maroc dans cette dynamique et d'analyser les spécificités de sa démarche. 
En 2007, lors du changement de gouvernement, il se crée le Ministère de l'Habitat, de l'Urbanisme et de l'Aménagement de l'Espace (MHUAE), doublé d'un Secrétariat d'Etat chargé du Développement Territorial. « Le regroupement des trois domaines stratégiques, urbanisme, habitat et développement territorial au sein d'un même département, confirme la volonté de l'Etat de donner une autre dimension à l'appréhension des problématiques socioéconomiques du pays en les « repositionnant » par rapport à leur cadre territorial.» (Adidi, A. 2010)

Ce retour au territoire est de nature à redynamiser l'activité économique mais surtout à retrouver la vision du territoire comme espace d'une communauté vivante contribuant efficacement à la cohésion sociale. (Sedjari, A., 1999) .Pour cela, il faut :

$\checkmark$ Imaginer des idées nouvelles pour la gestion des territoires;

$\checkmark$ Des approches novatrices d'aménagement du territoire ;

$\checkmark$ Des programmes ambitieux pour réduire les écarts entre les différents territoires.

Ainsi, cette nouvelle conception du développement met le territoire au centre de l'action publique. Plusieurs axes stratégiques en matière de développement territorial ont été déclinés. La promotion des réseaux des entreprises pour favoriser la coordination et les coopérations inter-entrepreneuriales, le développement des outils de veille et de l'innovation et la construction d'un environnement institutionnel favorable pour développer un nouveau mode de gouvernance capable de réunir les acteurs locaux avec les compétences nécessaires autour d'une vision commune.

\subsection{Les Systèmes productifs locaux : une forme d'organisation productive territorialisée.}

Les Systèmes Productifs Locaux (SPL) portent une nouvelle approche qui conçoit le territoire comme un moteur de développement économique favorisant la mise en œuvre d'une intelligence collective réunissant les acteurs locaux autour des projets communs. Ainsi, la démarche SPL conduit à une nouvelle conception du développement dans laquelle le territoire doit devenir la cible de l'action publique, l'acteur central étant désormais l'entreprise et les opérateurs économiques. En effet, chaque territoire, selon ses ressources, ses potentialités propres devrait contribuer au développement de l'activité productive nationale en s'appuyant sur une logique de réseaux qui fait de la solidarité entre les territoires une base pour le développement durable.

Au Maroc, plusieurs projets ont été entrepris par des départements ministériels .En effet, le Ministère chargé de l'industrie avait enclenché en 1997, en partenariat avec I'ONUDI et avec l'appui financier de l'Italie, un projet portant sur les stratégies pour la promotion des réseaux de PMI compétitifs et innovateurs qui contribueront à un développement industriel durable dans le cadre d'une dynamique de développement local. Ainsi, trois filières pilotes ont été choisies en raison de leur important potentiel de production, de leur vocation à l'export et de l'existence d'un intégrateur, dans la perspective d'étendre l'expérience à d'autres secteurs d'activités ; il s'agit des secteurs du cuir à Fès, de la poterie à Safi et de la marqueterie (travail du bois de thuya) à Essaouira.

Dans le même sens, le Ministère du Commerce Extérieur a lancé, toujours dans le cadre du même projet avec I'ONUDI, une initiative visant à encourager les opérateurs économiques, en particulier les PME/PMI, à se regrouper au sein de consortiums d'exportation dans le but d'améliorer leur accessibilité aux marchés extérieurs. Ainsi, tenant compte de l'expérience réussie de création et de développement des consortiums d'exportation durant la période 2003-2010 (21 consortiums d'exportation juridiquement créés), l'Etat compte donner un nouvel élan à ces groupements à travers la mise en place d'un programme d'appui renforcé d'un nouveau schéma de gouvernance. Ce programme baptisé « Export Synergia » est un instrument d'encouragement destiné à soutenir les consortiums d'exportation durant leur phase de démarrage à travers un accompagnement pour la réalisation des actions visant leur développement à l'international.

En 2002, le Ministère de l'Aménagement du Territoire, de l'Eau et de l'Environnement, a lancé en en partenariat avec l'Université Pierre Mendés (Grenoble), une étude sur le développement des bassins d'emploi (cas des SPL au Maroc). Cette étude a permis l'identification de 10 cas de Système Productifs Localisés (Courlet,C. 2006) potentiels II s'agit de :

$\checkmark$ Tanger (textile-habillement),

$\checkmark$ Casablanca (mécanique),

$\checkmark$ Casablanca (textile-habillement),

$\checkmark$ Casablanca (technologies de l'information),

$\checkmark$ Souss Massa (agriculture intensive),

$\checkmark$ Souss Massa (agriculture extensive),

$\checkmark$ Fès (artisanat),

$\checkmark$ Guercif (textile habillement), 
$\checkmark$ Guercif (oléiculture),

$\checkmark$ Arfoud (tourisme).

Cette identification conduit à penser que les conditions d'une coopération entre les acteurs d'un territoire existent effectivement. On constate cependant que sur les deux dimensions principales, à savoir l'engagement sans nuances des pouvoirs publics et l'engagement du monde économique, de grands progrès restent à faire. Des progrès dans cette direction font partie des objectifs des responsables au niveau national. Mais il est clair que la proximité géographique des entreprises peut aider amplement à accélérer ces progrès, à condition que les acteurs économiques concernés au niveau local, les intègrent dans leur stratégie.

La réalité marocaine présente plusieurs concentrations sectorielles et géographiques d'entreprises (systèmes productifs locaux). Cependant, la simple concentration d'entreprises opérant dans le même secteur n'est nullement un gage de succès. En effet, le SPL ne peut être "clés en main », il doit se construire progressivement à partir des habitants et des groupes sociaux en présence de leur histoire et de leur culture, et il doit être capable de s'enraciner et de s'ancrer dans les territoires.

Et pour pouvoir comprendre les tendances mondiales et modifier leurs stratégies, les SPL doivent observer ce qui se passe ailleurs. II est indispensable qu'ils établissent des contacts avec l'extérieur, qu'ils participent à des chaînes de production internationales et qu'ils s'exposent aux meilleures pratiques internationales pour ne pas rester isolés et risquer l'implosion.

\subsection{L’Agenda 21 local : un outil privilégié pour la mise en œuvre du développement durable}

Le programme «Agendas 21 locaux pour la promotion de l'environnement et du développement durable en milieu urbain», se basant sur une approche participative, est appliqué dans les villes d'Agadir, Marrakech et Meknès, sur la base d'une première expérience à Essaouira. Ce dernier s'élargit aujourd'hui à d'autres villes et régions, consolidant de ce fait, le mouvement d'expansion et d'enracinement de cette nouvelle approche du développement à l'échelle locale. L'intérêt particulier que suscite ce programme découle de sa finalité qui se ramène à instaurer un processus d'ancrage et de promotion de la bonne gouvernance locale et du développement durable d'une cité.

Ce Programme initié, avec le soutien financier du Ministère marocain de l'aménagement du territoire, de l'eau et de l'environnement et le concours du PNUD, entre 2001-2004, s'est développé depuis par :

$\checkmark$ Une extension au niveau de la région Marrakech-Tensift Al Haouz,

$\checkmark$ Un programme au niveau de la ville d'El Hajeb,

$\checkmark$ Un programme relatif à la province d'El Hoceima,

$\checkmark$ Et par un autre programme qui a concerné cinq provinces du Sud.

Les programmes agendas 21 locaux visent à soutenir le développement d'une capacité d'appui aux municipalités et communes pour la préparation et la mise en œuvre d'Agendas 21 locaux. II s'agit notamment de mettre en œuvre les instruments appropriés en termes de planification stratégique participative, de renforcement des capacités des acteurs locaux, de protection de l'environnement et de conduite de projets à caractère démonstratif.

II est certain que la concrétisation de cette approche est une œuvre de longue haleine. En effet, Si les efforts d'implication et de mobilisation des acteurs locaux ont permis d'atteindre des résultats éloquents pour certains programmes, il est loin d'être satisfaisant dans certaines villes. Ainsi, des partenaires directs du Programme qui sont habitués à des projets physiques et concrets leur permettant plus de visibilité, conçoivent mal un projet d'appui institutionnel dont les résultats sont, selon eux, très peu palpables. Il a été également remarqué que la participation aux activités du programme est timide de la part de certains élus qui font partie de l'opposition dans le conseil municipal malgré le fait que l'Agenda 21 offre un espace de débat et d'échange à l'ensemble des forces vives de la cité.

\subsection{Programme de Développement Durable des Oasis du Tafilalet}

Le Programme de Développement Territorial Durable des Oasis du Tafilalet (POT) se situe dans le cadre du plan d'action de la stratégie de la lutte contre la désertification, de la préservation de la diversité biologique et d'autres aspects liés à la gestion de l'environnement. En effet, conscient du problème de dégradation des oasis du Sud, le gouvernement marocain a effectué un diagnostic et élaboré une stratégie et un plan d'action, à la suite desquels ce programme a été préparé pour tenter la création d'une dynamique de développement durable dans les espaces oasiens. II intervient au niveau de 29 communes afin d'appuyer, valoriser et rendre au Tafilalet son apogée. 
Ce territoire dispose d'une richesse architecturale de grande qualité, qu'il faut sauvegarder, notamment les «Ksours» et «qasbahs», des palmeraies productives des meilleures dattes, des paysages fascinants et un système ingénieux de drainage des eaux souterraines (khettara) qui témoigne du savoir faire des hommes de Tafilalet.

Le POT a été mis en œuvre en 2006 par la Direction de l'Aménagement du Territoire, et il s'est renforcé en 2009, grâce à la contribution du Fonds de développement rural (FDR). Son objectif global étant l'élaboration d'un plan de développement territorial durable dans le Tafilalet en se basant sur les plans communaux et intercommunaux de développement. C'est un programme fédérateur puisqu'il implique plusieurs intervenants :

$\checkmark$ Le Ministère de l'Habitat, de l'Urbanisme et de l'Aménagement de l'Espace,

$\checkmark$ La région Meknès-Tafilalet,

$\checkmark$ La province d'Errachidia,

$\checkmark$ L'Office de mise en valeur agricole du Tafilalet,

$\checkmark$ L'ADS,

$\checkmark$ INRA,

$\checkmark$ Bailleurs de fonds internationaux : PNUD, Fonds français pour l'environnement mondial (FFEM), le Fonds mondial pour l'environnement,

$\checkmark$ la Principauté de Monaco,

$\checkmark$ I'ONG internationale CARI France.

Pour préserver sa culture, ses valeurs, ses traditions, son identité et son environnement le Maroc s'est engagé dans une démarche de développement touristique durable. A ce titre, une stratégie pour le développement de l'écotourisme a été élaborée en 2007 pour positionner la Destination Ecotouristique des Oasis et du Désert du Tafilalet à travers un produit écotouristique phare et compétitif : la « Route du Majhoul », sa finalité est de promouvoir un tourisme durable dans la région, à travers une valorisation rationnelle et pérenne des ressources naturelles des Oasis du Tafilalet tout en intégrant une dimension de développement économique localisé. II s'agit d'initier plus particulièrement des actions liées à l'accueil avec des acteurs locaux, et de privilégier des retombées directes pour les communautés locales impliquées.

Ce produit écotouristique permet, à travers un circuit, de découverir plus de 100 variétés de dattes du Pays des oasis du Tafilalet, mais aussi de découvrir les produits de terroir en lien avec la production des dattes dans le Tafilalet : plats, mets, sucreries, jus, pattes de dattes, etc...

Le visiteur à travers plusieurs activités ludiques et récréatives va de site en site, d'oasis en oasis découvrir et apprendre ce qu'est une oasis, une Khettara, l'architecture de terre, etc.....

la Route du Mejhoul permet donc de structurer une filière agro-touristique pour la valorisation de la datte en mettant en réseau tous les acteurs concernés : hébergeurs, restaurateurs, associations, communes, agriculteurs, coopératives, producteurs, MINTOUR, ORMAVAT, ADS,...

Partenaires du Programme des Oasis de Tafilalet (POT), les gîtes el Khorbat à Tinjdad et Chez Pauline à Tadighoust obtiennent le label Clé Verte pour l'année 2010. Ce résultat est le fruit d'un processus d'encadrement mené par le Programme au profit de l'ensemble des gîteurs adhérents à l'APECTAF (Association des Promoteurs de l'Ecotourisme au Tafilalet).

Les objectifs du projet ne seront atteints qu'avec la mise en place d'outils techniques et pédagogiques. La mobilisation des ressources humaines et financières par une stratégie de communication adaptée, la capitalisation des bonnes pratiques et la réalisation des réajustements éventuels par un système de suivi évaluation efficace ainsi que la collecte, le traitement des données et la production de supports numérique et cartographique dans le cadre d'un système d'information géographique.

\section{Conclusion}

Personne ne peut se prononcer sur l'efficacité et la capacité des projets menés actuellement à accélérer les dynamiques du développement durable du territoire (Elkadiri,N., 2007). II est certain que la concrétisation de cette approche est une œuvre de longue haleine. Plusieurs raisons peuvent être citées :

$>$ Une tendance à la bureaucratisation des fonctions communales au détriment des missions de développement;

$>$ Les leviers de développement sont insuffisamment maitrisés et la perspective stratégique est souvent absente au profit des questions d'urgence et de court terme;

$>$ Les ONG locales manquent de moyens et de compétences; 
Des difficultés de coopération entre les acteurs,

$>$ Non-assimilation/appropriation des acteurs locaux (élus, services extérieurs, société civile...) des principes du DD.

Le processus d'élaboration des projets de développement territorial devient un enjeu en soi, car la mobilisation des acteurs ne se décrète pas et ne peut être «clés en main ». Elle se construit à travers une ingénierie de la participation des acteurs.

\section{Références}

Adidi, A. (2010). De l'aménagement du territoire au développement territorial : quelle transition et quelle articulation ?. Actes du colloque international Développement local et cohésion territoriale organisé à Rabat les 19 et 20 juin 2009 à la FSJES Souissi, Rabat.p 160.

Burgenmeier, B. (2007). Economie du développement durable, questions d'économie et de gestion. Editions de Bœck. p 38.

CMED. (1989). Notre avenir à tous. Editions du Fleuve, Montréal.

Courlet,C. (2006) . Territoire et développement économique au Maroc, le cas des systèmes productifs localisés. L'Harmattan. P 31.

Dion,M., Wolf,D. et al (2008) . Le développement durable : théories et applications au management. Dunod. p 96.

Elkadiri,N. (2007) . Eléments d'analyse sur le développement territorial : aspects théoriques et empiriques. L'Harmattan, Paris et Economie critique, Rabat.

Ferone,G., Debas, D. et Genin, A.S. (2004) . Ce que développement durable veut dire. Editions d'Organisation. p 6.

Gagnon, C. (2007). Définitions de l'Agenda 21e siècle local. Un outil intégré de planification du développement durable viable. Dans Gagnon, C. (Éd) et E, ARTH.

Pierron, J.P. (2009). Le développement durable. Ellipses Edition. p 52.

Sedjari, A. (1999). Aménagement du territoire et développement durable, quelle intermédiation ? . GRET. L'Harmattan. P 16.

Vandermotten, C. (2002). Le développement durable des territoires. Editions de l'Université de Bruxelles. p 45. 